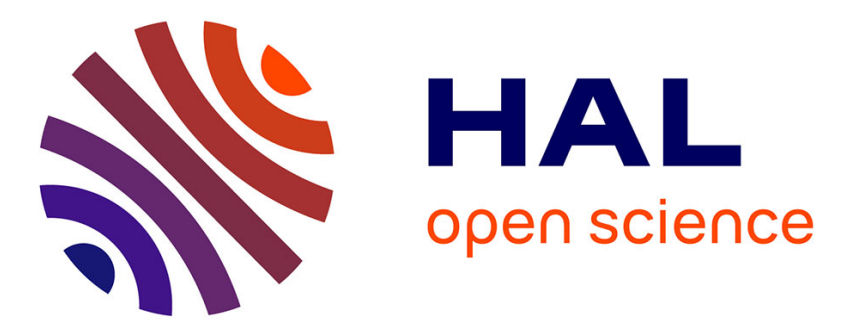

\title{
Role of surface plasmon in second harmonic generation from gold nanorods
}

Christophe Hubert, Laurent Billot, Pierre-Michel Adam, Renaud Bachelot, Pascal Royer, Johan Grand, Denis Gindre, Kokou Dorkenoo, A. Fort

\section{- To cite this version:}

Christophe Hubert, Laurent Billot, Pierre-Michel Adam, Renaud Bachelot, Pascal Royer, et al.. Role of surface plasmon in second harmonic generation from gold nanorods. Applied Physics Letters, 2007, 90 (18), pp.181105/1-3. 10.1063/1.2734503 . hal-00212190

\author{
HAL Id: hal-00212190 \\ https://hal.science/hal-00212190
}

Submitted on 12 Oct 2020

HAL is a multi-disciplinary open access archive for the deposit and dissemination of scientific research documents, whether they are published or not. The documents may come from teaching and research institutions in France or abroad, or from public or private research centers.
L'archive ouverte pluridisciplinaire HAL, est destinée au dépôt et à la diffusion de documents scientifiques de niveau recherche, publiés ou non, émanant des établissements d'enseignement et de recherche français ou étrangers, des laboratoires publics ou privés. 


\title{
${ }^{1}$ Role of surface plasmon in second harmonic generation from gold 2 nanorods
}

\author{
3 C. Hubert, ${ }^{\text {a) }}$ L. Billot, P.-M. Adam, R. Bachelot, and P. Royer \\ 4 Laboratoire de Nanotechnologie et d'Instrumentation Optique, Institut Charles Delaunay, CNRS-FRE 2848, \\ 5 Université de Technologie de Troyes, 12 rue Marie Curie, BP 2060, 10010 Troyes Cedex, France \\ $6 \quad$ J. Grand \\ $7 \quad$ Laboratoire ITODYS, UMR CNRS 7086, Université Paris 7-Denis Diderot, 1, rue Guy de la Brosse, F- \\ 875005 Paris, France \\ 9 \\ 10 \\ 11
}

12

13

14

15

16

17

18

19

20

22

The linear optical properties of metallic nanoparticles 23 (MNs) are dominated by collective oscillations of the con24 duction electrons. In particular, noble MNs present localized 25 surface plasmon resonances (LSPRs) that lead to a strong 26 absorption/scattering and local field enhancement near such 27 structures. ${ }^{1}$ The spectral position of these resonances de28 pends on the particles shape and size as well as on the nature 29 of the particle and the refractive index of the surrounding 30 medium. $^{2}$ Together with their linear properties, nonlinear op31 tical properties of metallic nanoparticles were studied and 32 appear promising for photonic applications. ${ }^{3}$ Because of 33 symmetry considerations, second harmonic generation 34 (SHG) is forbidden for centrosymmetric systems and thus 35 strongly depends on defects and small deviations from the 36 symmetric shape as well as from broken symmetry at inter37 faces. Nonlinear studies have been reported in the case of 38 metal colloids and tips through measurements of hyper39 Rayleigh scattering and SHG. ${ }^{4-6}$ SHG from nanostructures 40 with a low degree of symmetry and from noncentrosym41 metrical structures composed of symmetrically shaped par42 ticles was also investigated. $^{7-9}$ Polarization studies have 43 shown that SHG can be enhanced via resonant excitation of 44 LSPR (Refs. 9-11) of nanoparticles. More recently, SHG 45 measurements of planar symmetrical structures were 46 achieved using non-normal incidence illumination ${ }^{12}$ or by 47 looking at angles other than the illumination direction. ${ }^{10}$

48 So far, no spectral study of localized surface plasmons 49 on nanoparticles has been reported using SHG as a probe. In 50 this letter, we present a spectral study of SHG from periodic 51 arrays of gold nanorods. We demonstrate that SHG strongly

\footnotetext{
a) Author to whom correspondence should be addressed; Present address: Laboratoire Hubert Curien, UMR CNRS 5516, Université Jean Monnet, 18 rue Pr Benoît Lauras, 4200 Saint-Etienne, Cedex 2, France; electronic mail: christophe.hubert@univ-st-etienne.fr

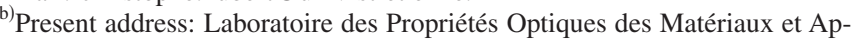
plications, UMR CNRS 6136, Université d'Angers, 2 Boulevard Lavoisier, F-49045 Angers Cedex, France.
}

depends on the incident light polarization direction and that $\mathbf{5 2}$ its excitation spectroscopy unambiguously evidences the role 53 of LSPR in the second harmonic signal enhancement. 54

Figure 1(a) shows the experimental setup used to gener- 55 ate and detect the second harmonic signals. A femtosecond $\mathbf{5 6}$ Ti:sapphire laser beam (100 fs, $80 \mathrm{MHz}$ repetition rate) is 57 focused onto the nanostructures using a $\times 20$ microscope ob- 58 jective into a wide laser spot of $1.3 \mu \mathrm{m}$ in diameter. The 59 pump wavelength can be tuned from 740 to $860 \mathrm{~nm}$. The 60

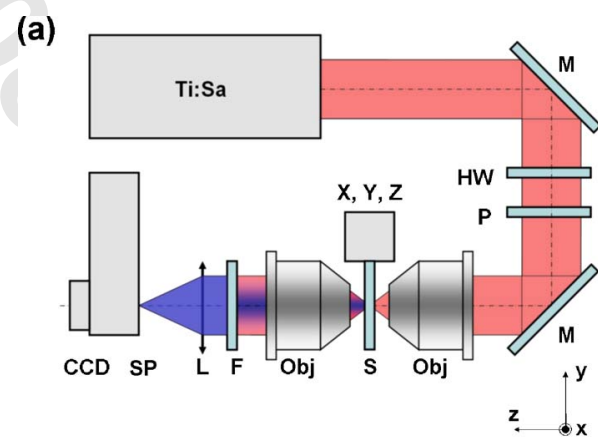

(b)

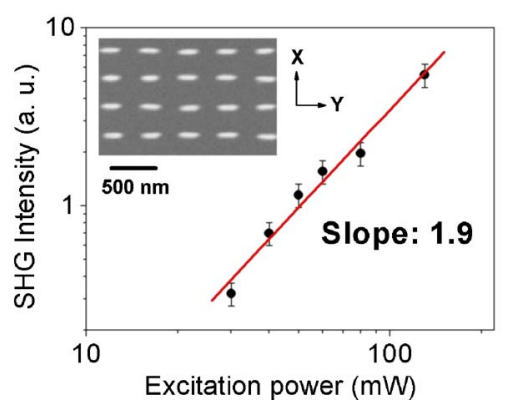

FIG. 1. (Color online) (a) Experimental setup. (b) Excitation power dependence of the detected SHG signal. The height, width, and length of the gold nanorods were equal to 60,50 , and $150 \mathrm{~nm}$, respectively. Irradiation wavelength was equal to $800 \mathrm{~nm}$ and incident polarization was parallel to the nanoparticle long axis. The insert shows a scanning electron microscope image of gold nanorods. $X$ and $Y$ directions are shown with arrows. 


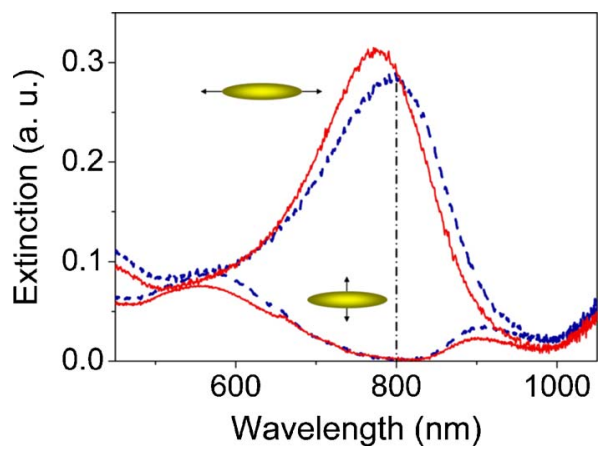

FIG. 2. (Color online) Extinction spectra from arrays of gold nanorods with long axis equal to $150 \mathrm{~nm}$ (solid curve) and $170 \mathrm{~nm}$ (dashed curve). The incident polarization used to record the spectra is schematized by arrows.

61 incident polarization direction and power are controlled with 62 a half-wave plate and a Glan-Taylor polarizer. The sample is 63 moved in the focal plane of the first objective microscope 64 using three-dimensional microdisplacement. The SHG signal 65 is collected in transmission through the sample with a second $66 \times 20$ microscope, and a BG 39 Schott filter is used to elimi67 nate the fundamental beam. The SHG spectra are measured 68 by a spectrometer and a charge coupled device. The insert in 69 Fig. 1(b) shows a scanning electron microscope image of the 70 nanostructures fabricated by electron beam lithography 71 through the lift-off method. ${ }^{6}$ Spacing between the ellipses is 72 kept constant and equal to $200 \mathrm{~nm}$ both in the $x$ and $y$ direc73 tions. We chose this edge to edge distance so that no strong 74 near-field coupling nor any grating effect (i.e., far-field cou75 pling) are observed in the extinction spectra. Because of this, 76 and also because of the homogeneity in size and shape of the 77 patterned area, we trust the extinction spectra to reflect the 78 optical properties of one particle and believe that we are 79 probing a single particle response. The long axis length is 80 varied from 150 to $190 \mathrm{~nm}$, whereas the short axis and the 81 height of the nanostructures are equal to 50 and $60 \mathrm{~nm}$, re82 spectively. Figure 1(b) shows the excitation power depen83 dence of the SHG signals measured on gold nanorods by 84 fitting a Lorentzian to a series of spectra. The linear fit of the 85 variation of the emitted signal versus pump power in a loga86 rithmic scale shows that the second harmonic signal has a 87 nearly quadratic dependence on the excitation intensity 88 (slope: 1.9), a characteristic of second order nonlinear pro89 cesses. The extinction spectra were recorded using a spec90 trometer coupled to a microscope by means of an optical 91 fiber.

92 Figure 2 shows the extinction spectra from arrays of na93 norod particles. The maxima that peak at 776 and $795 \mathrm{~nm}$ 94 correspond to nanoparticles with long axes of 150 and $95170 \mathrm{~nm}$, respectively, and are associated with the long axis 96 resonance mode of the nanostructures. As can be observed, 97 the peak of the LSPR associated with the nanorod long axis 98 is tuned to the laser wavelength at $800 \mathrm{~nm}$. The resonance 99 features in the extinction spectrum along the short axis at 100 wavelengths greater than $800 \mathrm{~nm}$ cannot be attributed to a 101 plasmon resonance and even though their origin has not been 102 clearly established yet, they do not reflect any particular 103 physical property of the nanoparticle themselves.

104 Figure 3(a) shows the typical spectra obtained when il105 luminating gold nanorods of $150 \mathrm{~nm}$ long axis with the fem106 tosecond $800 \mathrm{~nm}$ pump beam. The polarization angle $\theta$ of PROOFOCOPYin@idenAakser beam is defined with respect to the nanorod
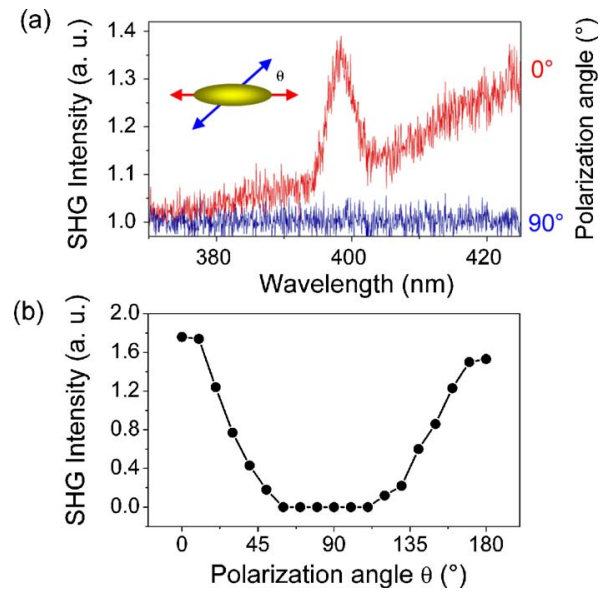

FIG. 3. (Color online) (a) Second harmonic spectra from arrays of $150 \mathrm{~nm}$ long axis gold nanorods. (b) Integrated second harmonic intensity from arrays of $150 \mathrm{~nm}$ long axis gold nanorods for different incident polarization angles $\theta$. The irradiation time and power used to record the spectra were equal to $5 \mathrm{~s}$ and $50 \mathrm{~mW}$, respectively. Irradiation wavelength was set to $800 \mathrm{~nm}$.

long axis, as indicated in the insert of Fig. 3(a). The maxi- 108 mum intensity of the second harmonic generation signal is 109 obtained when the incident light polarization direction is par- 110 allel to the nanorod long axis $\left(\theta=0^{\circ}\right)$, i.e., when the LSPR 111 associated with the long axis of the nanoparticles is excited. 112 On the contrary, when the polarization angle is equal to $90^{\circ}, 113$ no second harmonic signal is detected. The spectrum corre- 114 sponding to $\theta=0^{\circ}$ contains, in addition to the SHG signal, 115 the beginning of a broad peak centered at a higher wave- 116 length falling outside of our detection window. This corre- 117 sponds to the photoluminescence of the nanoparticles under 118 two-photon excitation and has been already observed. ${ }^{13,14} 119$

SHG intensity $I_{(2 \omega)}$ is proportional to the squared second 120 order nonlinear polarization $P^{(2)}$ and, in the case of metallic 121 nanoparticles, $I_{(2 \omega)}$ is proportional to ${ }^{15}$

$$
I_{(2 \omega)} \propto f_{(\omega)}^{4} f_{(2 \omega)}^{2} I_{(\omega)}^{2},
$$

where $f_{(\omega)}$ is the local field enhancement factor at the funda- 124 mental frequency, $f_{(2 \omega)}$ the local field enhancement factor at 125 the second harmonic frequency, and $I_{(\omega)}$ the pump beam in- 126 tensity. In the case of nanorods, $f_{(\omega)}$ can result from both 127 LSPR and off-resonance electromagnetic singularities ${ }^{16} 128$ (lightning rod effect). On the other hand, $f_{(2 \omega)}$ is expected to 129 result only from lightning rod effects because no resonance 130 is observable for these particles at such a wavelength due to 131 gold interband transitions. Figure 3(b) shows the influence of 132 the incident polarization on the second harmonic signal. The 133 second harmonic intensity is calculated by fitting each spec- 134 trum with a Lorentzian intensity distribution and then inte- 135 grating it. The second harmonic intensity $I_{(2 \omega)}$ is found to 136 strongly depend on the incident light polarization direction, 137 which indicates that the SHG is resonantly enhanced by 138 LSPR in nanoparticles preferentially excited when the light 139 polarization is oriented along the long axis of the nanorods. 140 Additionally, this polarization allows electromagnetic singu- 141 larities to be excited at the rod extremities. Given the poly- 142 crystalline nature of the samples, which is found to be glo- 143 bally isotropic, the orientational dependence of the frequency 144 doubling cannot be related to crystal structure. 

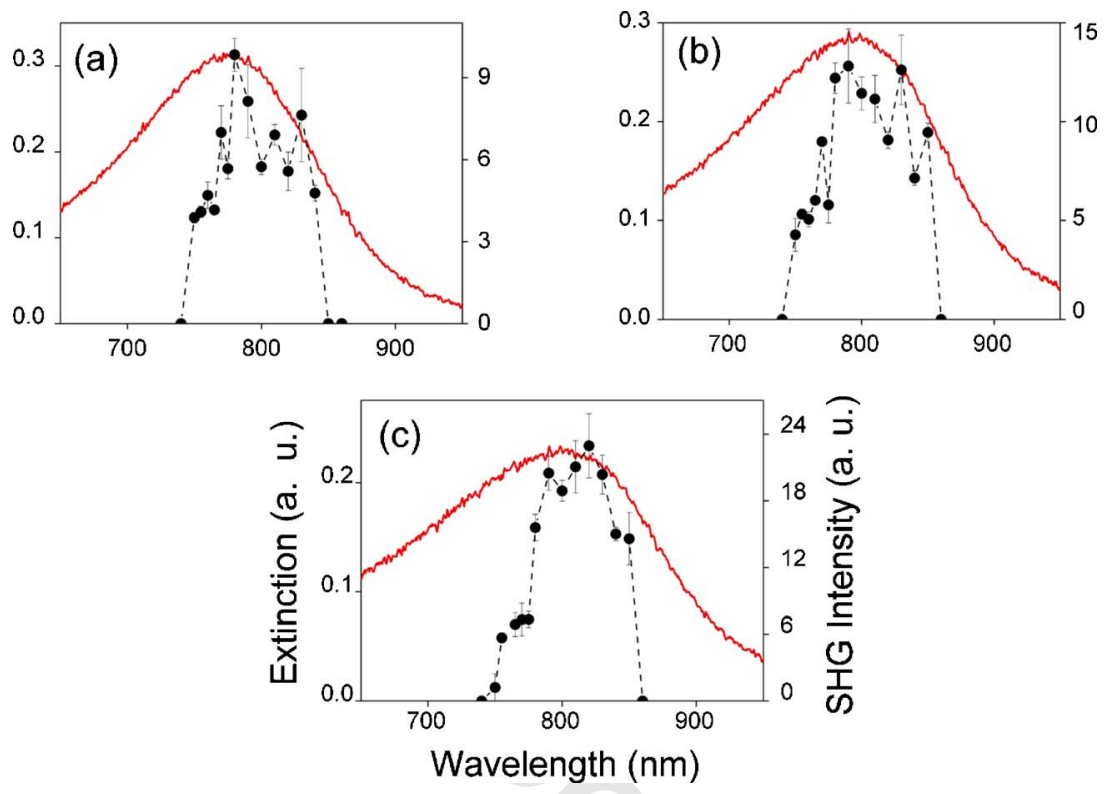

FIG. 4. (Color online) Second harmonic generation enhancement (circles) from arrays of gold nanorods with (a) $150 \mathrm{~nm}$, (b) $170 \mathrm{~nm}$, and (c) $190 \mathrm{~nm}$ long axis (the dashed line serves as a guide for the eyes). The extinction spectrum (solid line) is shown for comparison. The irradiation time and power used to record the second harmonic signal were equal to $5 \mathrm{~s}$ and $50 \mathrm{~mW}$, respectively. The incident polarization was set parallel to the nanoparticle long axis.

146

The results presented in Fig. 3 suggest the possible in147 fluence of plasmon resonance on second harmonic genera148 tion from the nanostructures. In Fig. 4, the study of the in149 fluence of the irradiation wavelength on the second harmonic 150 generation process confirms the role of the plasmon reso151 nance. Results from excitation spectroscopy of SHG from 152 150, 170, and $190 \mathrm{~nm}$ long axis gold nanorods are presented 153 in Fig. 4. The light polarization used to illuminate the struc154 tures and to record the extinction spectra is oriented parallel 155 to the nanorod long axis in order to maximize the SHG sig156 nal. It can be observed that whatever the long axis size, the 157 second harmonic signal follows the extinction spectrum 158 (solid line) of the nanoparticles and thus clearly demon159 strates the role of the LSPR in the SHG process. At the 160 half-width, the extinction spectrum peak is broader than the 161 one corresponding to the SHG intensity. This can be ex162 plained by the fact that the second harmonic signal is much 163 more sensitive to the field enhancement than extinction. In164 deed, nonlinear processes are particularly sensitive to these 165 local resonances due to their quadratic dependence on the 166 intensity. According to Eq. (1), $I_{(2 \omega)}$ is proportional to the 167 fourth power of the field enhancement at the fundamental 168 frequency, which originates from LSPR excitation. A small 169 variation in the plasmon resonance intensity thus leads to 170 strong variations in second harmonic intensity, as observed 171 in Fig. 4. The wavelength dependence studies may also sug172 gest that the second harmonic signal has a dipolar electric 173 origin rather than a quadripolar electric one. ${ }^{8}$ Although the 174 SHG is theoretically forbidden in centrosymmetrical sys175 tems, we make the assumption that, in our case, due to the 176 lithographic fabrication process, the nonlinear generation 177 process may arise from a deviation of the shape of the nano178 particles from that of a perfect symmetrical nanorod as well 179 as from the broken symmetry at the air-metal and metal180 substrate interfaces. Defects in the crystalline structure of 181 gold nanoparticles also have to be considered. Finally, it 182 should also be pointed out that due to the large range of wave 183 vectors produced by confined plasmon excitation, depolar184 ization effects can be induced; i.e., vertical component of the 185 near-field appears, making asymmetry discussion nontrivial. 186 In conclusion, our polarization and spectroscopic studies PROOFEC@exarloszhemAgnstrate that second harmonic generation from metallic nanoparticles strongly depends on their dipolar plas- 188 mon resonance. Resonance enhancement of the second har- 189 monic intensity has been observed while tuning the polariza- 190 tion of the pump beam from parallel to perpendicular to the 191 long axis of nanoparticles. We also observed a strong varia- 192 tion in the wavelength dependence of the second harmonic 193 generation intensity when the irradiation wavelength is tuned 194 towards the extinction peak. This further highlights the influ- 195 ence of the field enhancement from resonance plasmon exci- 196 tation in the second harmonic generation process from me- 197 tallic nanoparticles.

198

This work was supported by the Region Alsace and by 199 the Centre National de la Recherche Scientifique (CNRS). 200

${ }^{1}$ H. Raether, Surface Plasmons on Smooth and Rough Surfaces and on 201 Gratings (Springer, Berlin, 1988), $\mathbf{\square}, \mathbf{\square} .202$

${ }^{2}$ C. F. Bohren and D. R. Huffman, Absorption and Scattering of Light by 203 AQ: Small Particles (Wiley-Interscience, New York, 1983), ם, 口. 204 \#2

${ }^{3}$ J. Tominaga, C. Mihalcea, D. Büchel, F. Fukuda, T. Nakano, N. Atoda, H. 205

Fuhi, and T. Kikukawa, Appl. Phys. Lett. 78, 2417 (2001). 206

${ }^{4}$ J. Nappa, G. Revillod, I. Russier-Antoine, E. Benichou, C. Jonin, and P. F. 207 Brevet, Phys. Rev. B 71, 165407 (2005). 208

${ }^{5}$ E. C. Hao, G. C. Schatz, R. C. Johnson, and J. T. Hupp, J. Chem. Phys. 209 117, 5963 (2002).

${ }^{6}$ A. Bouhelier, M. Beversluis, A. Hartschuh, and L. Novotny, Phys. Rev. 211

Lett. 90, 013903 (2003).

${ }^{7}$ B. K. Canfield, S. Kujala, K. Jefimovs, T. Vallius, J. Turunen, and M. 213 Kauranen, J. Opt. A, Pure Appl. Opt. 7, S110 (2005).

${ }^{8}$ A. M. Moran, J. Sung, E. M. Hicks, R. P. Van Duyne, and K. G. Spears, J. 215 Phys. Chem. B 109, 4501 (2005).

${ }^{9}$ B. Lamprecht, A. Leitner, and F. R. Aussenegg, Appl. Phys. B: Lasers 217 Opt. 68, 419 (1999).

${ }^{10}$ M. D. McMahon, R. Lopez, R. F. Haglund, Jr, E. A. Ray, and P. H. 219 Bunton, Phys. Rev. B 73, 041401 (2006). 220

${ }^{11}$ R. A. Farrer, F. L. Butterfield, V. W. Chen, and J. T. Fourkas, Nano Lett. 221 6, 1139 (2005).

${ }^{12}$ A. Podlipensky, J. Lange, G. Seifert, H. Graener, and I. Cravetchi, Opt. 223 Lett. 28, 716 (2003).

${ }^{13}$ M. R. Beversluis, A. Bouhelier, and L. Novotny, Phys. Rev. B 68, 115433225 (2003).

${ }^{14}$ A. Bouhelier, R. Bachelot, G. Lerondel, S. Kostcheev, P. Royer, and G. P. 227 Wiederrecht, Phys. Rev. Lett. 95, 267405 (2005).

${ }^{15}$ A. Wokaun, in Solid State Physics, edited by H. Ehrenreich, T. Thurnbull, 229 and F. Seitz (Academic, New York, 1984), Vol. 38, p. 223.

${ }^{16}$ J. Van Bladel, Singular Electromagnetic Fields and Sources (IEEE, Ox- 231 ford, 1995), 
AUTHOR QUERIES — 108717APL

\#1 Au: Pls. check change of " $\square$ " to " $\mu$."

\#2 Au: Pls. supply volume \& page numbers for Refs. 1, 2, \& 16. 